\title{
Angular Distribution of N-Doped Carbon Nanotubes in Alumina Membrane Channels: A High-Energy X-ray Diffraction Study
}

\author{
L. Hawelek ${ }^{a}$, A. Brodka ${ }^{a}$, J.C. Dore ${ }^{b}$, V. Honkimäki ${ }^{c}$, T. Kyotani $^{d}$, \\ Q.H. YANG ${ }^{e}$ AND A. BURIAN ${ }^{a}$ \\ ${ }^{a}$ A. Chełkowski Institute of Physics, University of Silesia, Uniwersytecka 4, 40-007 Katowice, Poland \\ ${ }^{b}$ School of Physical Sciences, University of Kent, Canterbury, CT2 7NR, UK \\ ${ }^{c}$ European Synchrotron Radiation Facility, BP 220, 38043 Grenoble, France \\ ${ }^{d}$ Institute of Multidisciplinary Research and Advanced Material, Tohoku University \\ Katahira, Sendai 980-88577, Japan \\ ${ }^{e}$ School of Chemical Engineering and Technology, Tianjin University \\ 92 Weijin Road, Tianjin 300072, China
}

\begin{abstract}
An alignment study of multi-wall N-doped carbon nanotubes prepared by a template pyrolytic carbon deposition method inside channels of an alumina membrane has been performed using high-energy X-ray diffraction on the ID15B beamline at the European Synchrotron Radiation Facility (ESRF, Grenoble). The two-dimensional diffraction pattern of the deposited carbon nanotubes, recorded directly, within the alumina membrane, using an image plate detector, exhibits two non-continuous arcs corresponding to the 002 graphitic reflection. The following values of the angle between the axis of the carbon nanotubes lying along the membrane channels and the incident beam were taken for five positions: $0^{\circ}, 30^{\circ}, 45^{\circ}, 60^{\circ}$ and $90^{\circ}$. The anisotropic scattering distribution of the two-dimensional patterns indicates an orientational alignment of the nanotubes. The one-dimensional intensity patterns obtained by scanning around the circumference of the (002) ring have allowed an estimation of the angular distribution of the nanotubes axes.
\end{abstract}

PACS numbers: 61.05.cp, 61.48.De

\section{Introduction}

Since the discovery of carbon nanotubes (CNTs) by Iijima in 1991 [1], a number of studies on CNTs and related nanostructures have been carried out. The promising mechanical and electronic properties of these structures have led to several applications, including nanoelectronic devices [2], probe tips for scanning probe microscopy [3], and field emitter arrays [4]. Furthermore, it has been proved that CNTs have higher conductivity than graphite. Doping CNTs with heteroatoms could be particularly of interest for tuning the surface and electronic properties. Doping with nitrogen leads to an increase in the conductivity due to the raising of the Fermi level towards the conduction band [5, 6]. Various methods have been developed for the synthesis of the doped nanotubes: arc discharge, laser ablation, pyrolysis, substitution reaction and chemical vapor deposition (CVD) methods. The template synthesis method has been widely used for nanostructured materials [7], which involves the synthesis of the desired material within the pores of a membrane to generate CNTs with uniform di- ameter. The combination of the template synthesis and the CVD method to obtain highly-aligned, uniform, hollow and open-ended CNTs with diameters from 20 to $200 \mathrm{~nm}$ has been previously reported by Che et al. [8] and Kyotani et al. [9]. Among all the methods of preparation of CNTs, the template synthesis method has the advantage of controlling morphology, composition and the size of the nanotubes [10]. Two different synthesis methods have been used to produce the nitrogen doped carbon nanotubes (N-CNTs) inside alumina membrane channels. In the first one, the nitrogen-containing carbon nanotubes are prepared by the use of a liquid-phase impregnated template membrane with a polymer such as polyacrylonitrile [11], polypyrrole [12] or polyvinylpyrrolidone [13]. In the second method, pyrolytic carbon deposition from the thermal decomposition of acetonitrile at high temperature is used. In the present work the $\mathrm{N}-\mathrm{CNTs}$ with diameters which fall within the range of 25-30 nm (the diameter of the channels) and length within the range 50-100 $\mu \mathrm{m}$, were studied. The microchannels are perpendicular to the anodic aluminum oxide (AAO) membrane surface. A detailed knowledge 
of the structure of such fabricated N-CNTs is particularly necessary from the point of view of their future applications.

The atomic structure of the N-CNTs has been conventionally investigated using high-resolution transmission electron microscopy (HRTEM), scanning electron microscopy (SEM), atomic force or scanning tunneling microscopy (AFM, STM), electron diffraction (ED), Raman, IR and X-ray photoelectron (XPS) spectroscopic techniques. Such techniques give no information on the orientational characteristics, because they are made after removal of the template by chemical treatment. As was observed, the tubes became separated from each other and may be bent [14] due to the etching process. Therefore, it is desirable to study the template N-CNTs as deposited in the alumina membrane channels in order to check their alignment without the chemical removal of the template. Burian et al. [15] have used modern X-ray diffraction methods to obtain detailed information on the structure and orientation of the undoped CNTs deposited inside the membrane channels using a CVD method. In the present paper it is shown how the anisotropic scattering distribution of the two-dimensional pattern can be used to obtain information about the alignment of the $\mathrm{N}$-doped carbon nanotubes.

\section{Experimental}

The N-doped CNTs studied in the present work were deposited inside the parallel and straight nanochannels in an AAO film. A thickness of the AAO film was about $70 \mu \mathrm{m}$ and a channel diameter was $30 \mathrm{~nm}$. The thermal chemical vapour deposition technique was used under an acetonitrile vapour flow $\left(4.2 \%\right.$ in $\left.\mathrm{N}_{2}\right)$ at $800{ }^{\circ} \mathrm{C}$ for $5 \mathrm{~h}$ to obtain the N-doped CNTs. The decomposition of acetonitrile leads to the uniform coating of a N-doped carbon layer on the inner walls of the AAO nanochannels. The detailed description of the template synthesis of N-CNTs has been previously reported in $[16,17]$ and is not repeated here.

X-ray diffraction measurements were carried out on the ID15B beam line at the European Synchrotron Radiation Facility, Grenoble, France. An incident beam-energy of $95.4 \mathrm{keV}$, corresponding to a wavelength of $0.13 \AA$, was used to record two-dimensional diffraction patterns using an image plate as a two-dimensional detector. The sample with area of about $1 \mathrm{~cm}^{2}$ was chosen for measurements. The sample to detector distance was $260.2 \mathrm{~mm}$ and the beam width at the sample was approximately $300 \mu \mathrm{m}$. The MAR 2300 on-line image-plate detection system consists of $2300 \times 2300$ pixels, each of $0.15 \mathrm{~mm}$ in size. The $2 \mathrm{D}$ diffraction images were displayed and analyzed using the software Fit2D [18, 19]. The exposures were taken with the membrane channels positioned at $0^{\circ}, 30^{\circ}, 45^{\circ}, 60^{\circ}$ and $90^{\circ}$ angles between the axis of the carbon nanotubes and the incident beam. The additional exposure was made for the powdered sample of the N-CNTs, after removal of the AAO membrane by chemical treatment. The powdered sample was mounted in a Lindemann glass capillary on the sample holder and the intensity was measured as a function of the scattering vector $K(K=4 \pi \sin \theta / \lambda$, where $2 \theta$ is the scattering angle and $\lambda$ is the wavelength) were obtained up to $K_{\max }=24 \AA^{-1}$. The experimental procedures are described in detail in our previous papers [20, 21].

\section{Results and discussion}

The recorded $2 \mathrm{D}$ diffraction pattern for the sample of the N-doped multi-walled CNTs within the AAO membrane for five angle values between the incident beam and the membrane channels (or the nanotubes axis) and for the pure powdered N-CNTs sample are shown in Fig. 1. In the A-D (Fig. 1) positions of the membrane at $90^{\circ}, 60^{\circ}, 45^{\circ}$ and $30^{\circ}$ between the nanotube axes and the incident beam, the diffraction patterns exhibit an anisotropy in the 2D intensity distribution, however, when the membrane channels are parallel to the incident beam (i.e. the nanotubes axes are parallel to the incident beam), the complete Debye-Scherrer intensity ring is observed (Fig. 1E). In Fig. 1F the 2D diffraction pattern for the powdered sample is shown for comparison. In $\mathrm{A}-\mathrm{E}$ recorded diffraction patterns the remaining sharp features are believed to be due to partial crystallization of the alumina membrane during the CVD process. Similar observation has been already made for the undoped samples as described in [15]. Figure 2 gives a schematic drawing of the experiment when the nanotubes axes are perpendicular (Fig. 2A) and parallel (Fig. 2B) to the incident beam.

In Fig. 3, the white dotted circle marks the circle on which two arcs, corresponding to the 002 graphitic reflection, are seen. Such reflections arise from the multilayer structure of the N-CNTs and give an indication of the degree of alignment of the nanotubes. If the nanotube axes are perfectly perpendicular to the incident beam, these reflections would produce two sharp spots on the vertical axis of the image plate detector, but it can be seen that two arcs are visible on the dotted circle, which indicates a variation in the alignment. In Fig. 4 the intensity measured along the dotted circle shown in Fig. 3 is presented as a function of the azimuthal angle $\Psi$ in the range of $0-360^{\circ}$. For the 002 graphitic peaks, a non-linear Gaussian fitting procedure was used to estimate distribution of orientation of the nanotubes axes. For both arc-like reflections the Gaussian distributions with the parameters of the standard deviation, $\sigma$, and the total area, $A$, under the curve, match the experimental intensity profiles very well. The normal Gaussian probability distribution function is described by

$$
P(\Psi)=\frac{A}{\sigma \sqrt{2 \pi}} \exp \left(-\frac{\left(\Psi-\Psi_{0}\right)^{2}}{2 \sigma^{2}}\right),
$$

where $\Psi_{0} 0$ is the center of the peak, and $\Psi$ in our cases is the azimuthal angle. From the standard deviation, $\sigma$, obtained from the fitting procedure, the full-width at half-maximum, FWHM, can be calculated according to the formula 


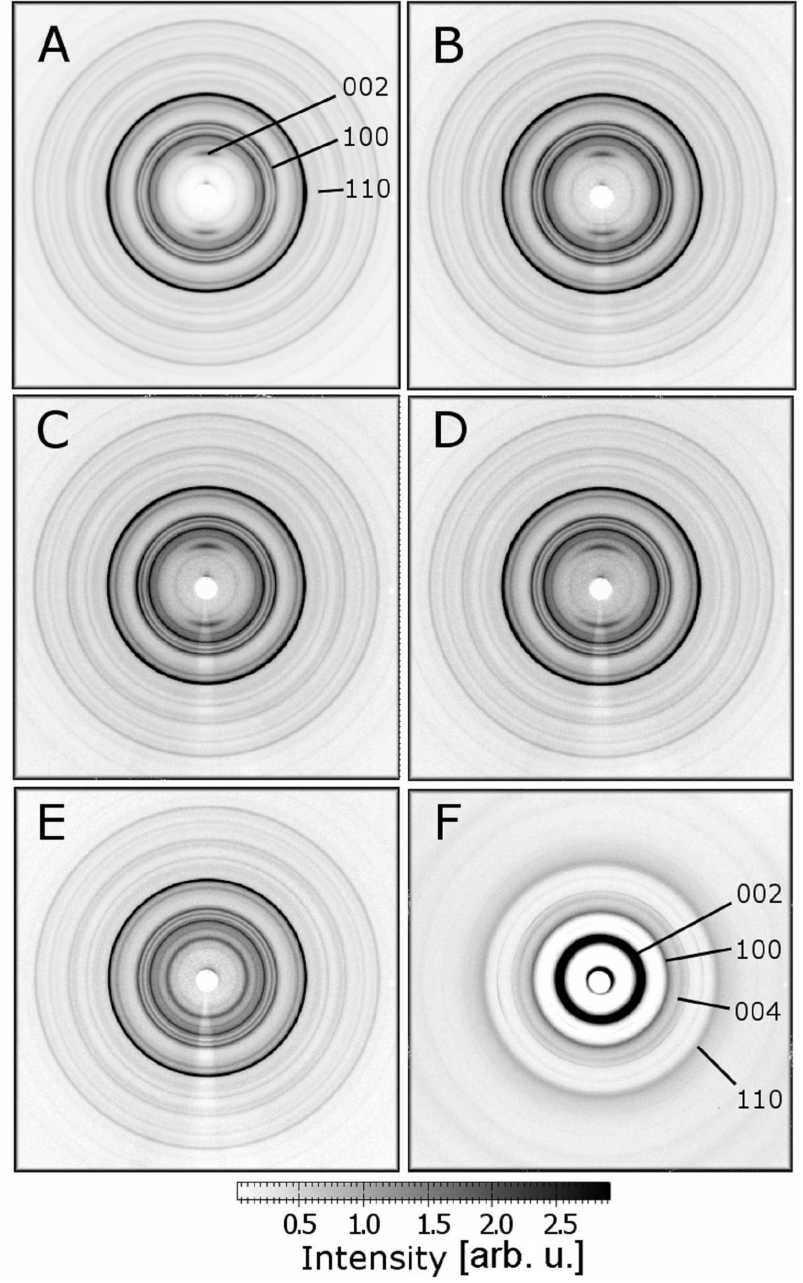

Fig. 1. The comparison of the 2D diffraction patterns of the N-CNTs grown inside the alumina membrane for five positions of membrane: $\mathrm{A}-\phi=90^{\circ}, \mathrm{B}-\phi=60^{\circ}$, $\mathrm{C}-\phi=45^{\circ}, \mathrm{D}-\phi=30^{\circ}$ and $\mathrm{E}-\phi=0^{\circ}$, and $\mathrm{F}$ for pure powdered sample. The indices of the N-CNTs are marked in parts A and F. Remaining Debye rings are due to the crystallized alumina membrane as described in the text.

$$
\mathrm{FWHM}=\sigma \sqrt{8 \ln 2} \cong 2.355 \sigma .
$$

For the perpendicular position with the axis of the carbon nanotubes parallel to the membrane channels and the incident beam, the azimuthal angle intensity variation for the 002 ring and the fitted Gaussian curve are shown in Fig. 4. For the intensity peak at $90^{\circ}$ (the top 002 arc) $\mathrm{FWHM}=33.8 \pm 0.1^{\circ}$ and for the peak at $270^{\circ}$ (the bottom 002 arc) the FWHM $=35.5 \pm 0.1^{\circ}$. The slight differences in both values of FWHM can be related to mis-orientation of the alumina membrane (and the N-CNTs) with respect to the goniometer centre and the incident beam. A similar procedure was applied to the membrane positions at $60^{\circ}, 45^{\circ}$ and $30^{\circ}$ with respect to the incident beam. The relevant plots of the experimental intensity and the Gaussian fits are shown in Fig. 5

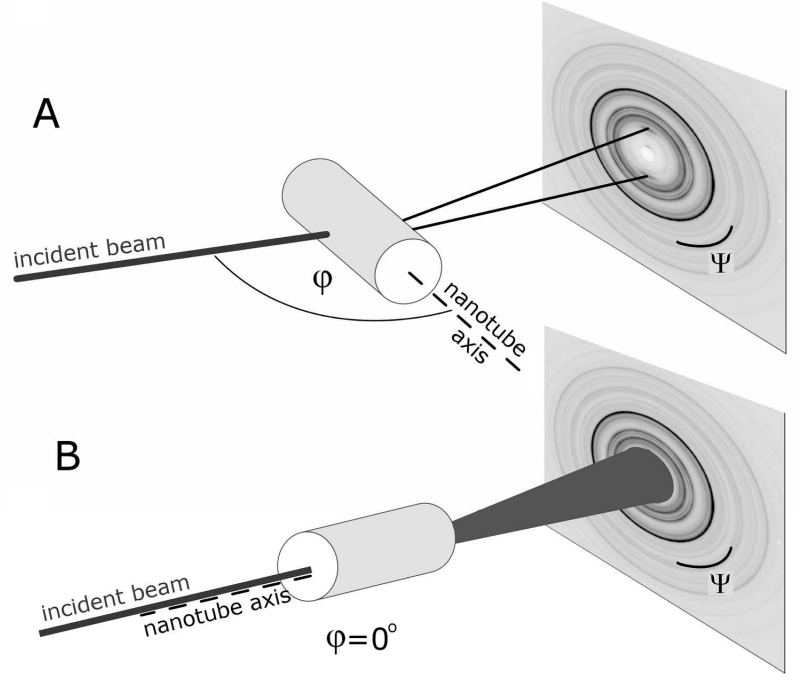

Fig. 2. Schematic drawing of the experiment when the nanotube axes are perpendicular $\phi=90^{\circ}$ (Fig. 2A) and parallel $\phi=0^{\circ}$ (Fig. 2B) to the incident beam.

together with the curves for the membrane position at $90^{\circ}$ for comparison. The values of the FWHMs are listed in Table and shown in Fig. 6 versus the orientation angle together with the fitted $30.9 / \cos \left(90^{\circ}-\phi\right)$ curve which describes in a reasonable approximation variation of the FWHM as the function of the orientation angle. For the position of the membrane at $0^{\circ}$ the intensity is distributed uniformly around the 002 circle. In this position a continuous Debye-Scherrer ring is observed as shown in Fig. 1E.

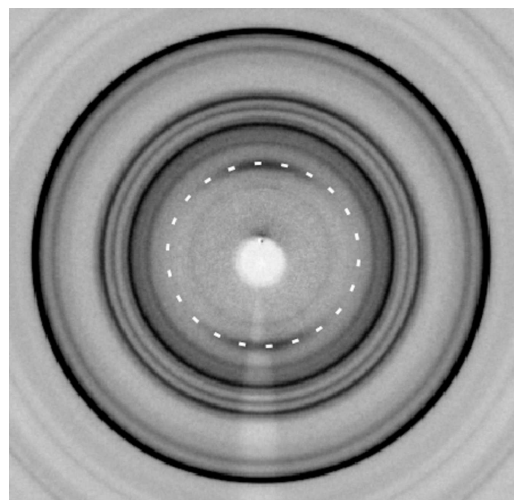

Fig. 3. The 2D diffraction pattern of the N-CNTs grown inside the alumina membrane. The white dotted circle marks the 002 graphite reflection ring.

The anisotropic variation of the diffraction intensity for the 002 peak, together with fitted Gaussian curve, shows the orientational variation of the nanotube axes with a FWHM value of approximately $30.9^{\circ}$, i.e. $\pm 15.45^{\circ}$. Within the Bragg approach the fragments of the nanotubes tangential to their surface and lying at the Bragg 


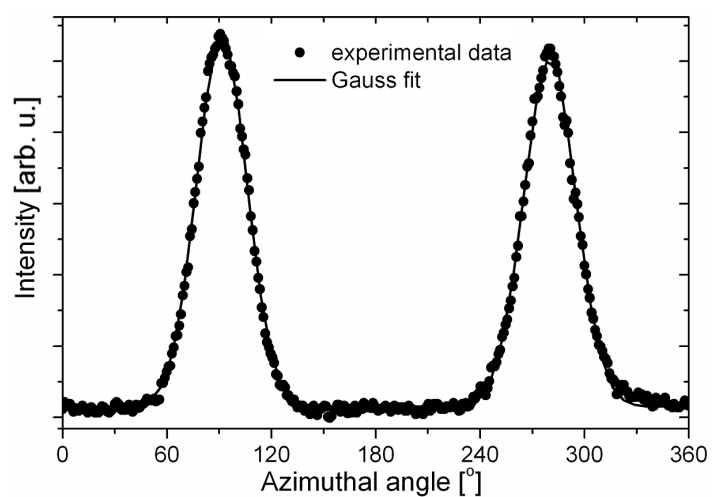

Fig. 4. Intensity variation of the 002 ring around the azimuthal angle together with the fitted Gaussian curves.

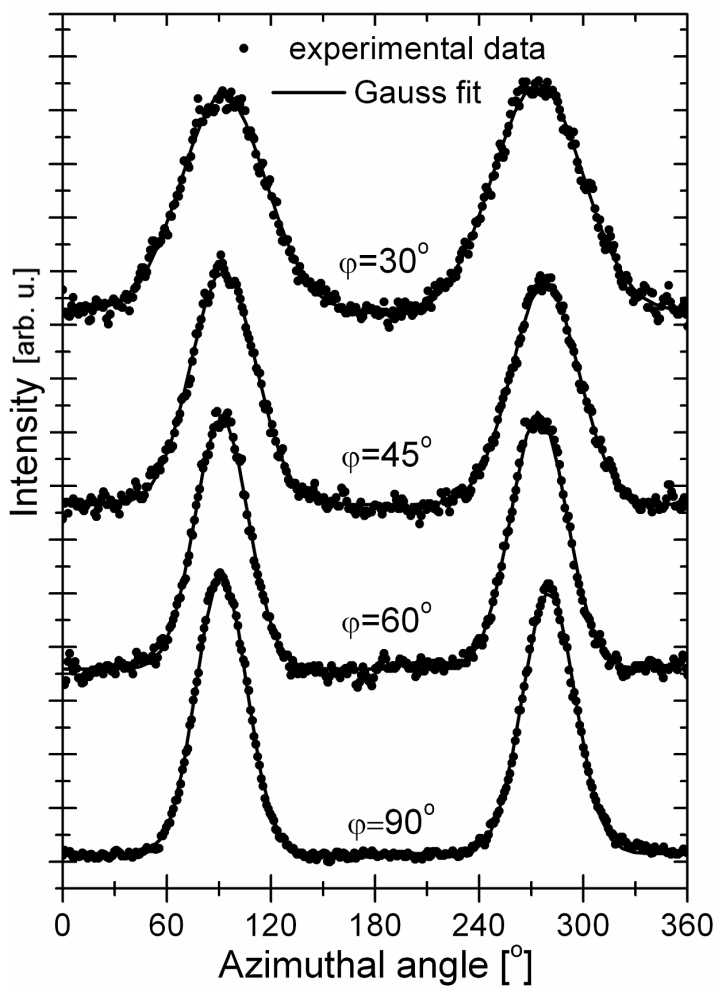

Fig. 5. The azimuthal angle intensity variation for the 002 ring for four positions of the axis of carbon nanotubes within the AAO membrane channels with respect to the incident beam $\left(30^{\circ}, 45^{\circ}, 60^{\circ}\right.$ and $\left.90^{\circ}\right)$.

angle with respect to the incident beam lead to occurrence of the 002 reflections. For the wavelength of $0.13 \AA$ the Bragg angle is approximately $1.1^{\circ}$, assuming the interlayer spacing of $3.4 \AA$. Therefore for the orientation of the membrane at $0^{\circ}$, a continuous Debye-Scherrer ring is observed. The distribution of the orientation of the nanotubes axes is characterized by the value of FWHM when the nanotubes axes are perpendicular to the incident beam.

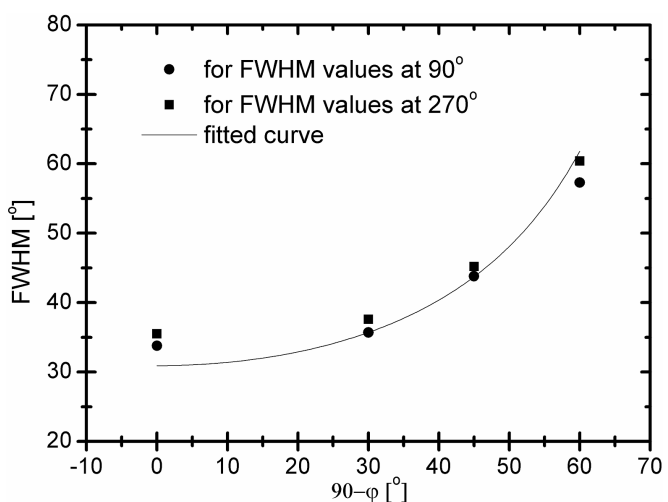

Fig. 6. The dependence FWHM on the AAO membrane positions $90^{\circ}-\phi$. The values of the FWHMs at $90^{\circ}$ and $270^{\circ}$ are shown as circles and squares, respectively.

TABLE

The comparison of calculated FWHM parameters for both arcs of the 002 reflection for four positions of the angle between the axis of carbon nanotubes within membrane channels and the incident beam $\left(\phi=30^{\circ}, 45^{\circ}, 60^{\circ}\right.$ and $\left.90^{\circ}\right)$.

\begin{tabular}{c|c|c}
\hline \hline$\phi\left[^{\circ}\right]$ & $\begin{array}{c}\text { FWHM at azimuthal } \\
\text { angle } 90^{\circ}\left[{ }^{\circ}\right]\end{array}$ & $\begin{array}{c}\text { FWHM at azimuthal } \\
\text { angle } 270^{\circ}\left[{ }^{\circ}\right]\end{array}$ \\
\hline 90 & $33.8 \pm 0.1$ & $35.5 \pm 0.1$ \\
60 & $35.7 \pm 0.2$ & $37.6 \pm 0.2$ \\
45 & $43.8 \pm 0.3$ & $45.2 \pm 0.3$ \\
30 & $57.3 \pm 0.4$ & $60.4 \pm 0.4$
\end{tabular}

The value of the orientational variation for the nanotubes suggests a more defective and curved structure for the investigated N-doped CNTs, that was also observed by Yang et al. [16]. Later analyses have revealed that $\mathrm{N}$ atoms substitute carbon atoms with a small portion in the pyridinic N. Previous studies also suggest that the local perturbation of the hexagonal structure may be generated by introducing pentagon-heptagon pairs into the CNTs as the Stone-Wales defects [22]. Such defects lead to a local curvature of the nanotubes surfaces and may contribute to local mis-orientation of their axes.

\section{Conclusions}

The present results demonstrate that the very well-collimated X-ray beam of the short-wavelength available at a third generation synchrotron source can be used for alignment studies of the $\mathrm{N}$-doped carbon nanotubes. The orientation effect observed for the 002 diffraction peak when the nanotubes axes are perpendicular to the incident beam gives information about the degree of alignment of the N-CNTs deposited inside the nanochannels of the AAO membrane, which can be estimated from broadening of two anisotropic arcs corresponding to this 
graphitic reflection. The present results are in agreement with the previous scanning electron microscopy observations reported in [17], which have shown that the investigated N-doped carbon nanotubes are straight. However, these observations were performed for the N-CNTs after removal of the template. In this paper the method which allows qualitative estimation of angular distribution of the N-CNTs inside the channels of the alumina membrane is proposed.

\section{References}

[1] S. Iijima, Nature 354, 56 (1991).

[2] S.T. Trans, A.R.M. Verscheren, C. Dekker, Nature 393, 49 (1998).

[3] S.S. Wong, E. Joselevich, A.T. Woolley, C.L. Cheung, C.M. Lieber, Nature 394, 52 (1998).

[4] S.S. Fan, M.G. Chapline, N.R. Franklin, T.W. Tombler, A.M. Cassell, H.J. Dai, Science 283, 512 (1999).

[5] M. Terrones, P.M. Ajayan, F. Banhart, X. Blase, D.L. Carroll, J.C. Charlier, R. Czerw, B. Foley, N. Grobert, R. Kamalakaran, P. Kohler-Rodlich, M. Ruhle, T. Seeger, H. Terrones, Appl. Phys. A Mater. 74, 355 (2002).

[6] R. Czerw, M. Terrones, J.C. Charlier, X. Blase, B. Foley, R. Lamalakaran, N. Grobert, H. Terrones, D. Tekleab, P.M. Ajayan, W. Blau, M. Ruehle, D.L. Carroll, Nano Lett. 9, 457 (2001).

[7] B. Rajesh, K. Ravindranathan, J.M. Thampi, N. Bonard, H.J. Xanthopoulos, B. Mathieu, B. Viswanathan, J. Phys. Chem B 107, 2701 (2003).
[8] G. Che, B.B. Lakshmi, C.R. Martin, E.R. Fisher, R.A. Ruoff, Chem. Mater. 10, 260 (1998).

[9] T. Kyotani, L.F. Tsai, A. Tomita, Chem. Mater. 7, 1427 (1996).

[10] R. Che, L.M. Peng, Q. Chen, X.F. Duan, Z.N. Gu, Appl. Phys. Lett. 82, 331 (2003).

[11] R.V. Parthasarathy, K.L.N. Phani, C.R. Martin, Adv. Mater. 7, 896 (1995).

[12] J. Jang, J.H. Oh, Chem. Commun. 2004, 882 (2004).

[13] T. Maiyalagan, B. Viswanathan, Mater. Chem. Phys. 93, 291 (2005).

[14] J.S. Lee, G.H. Gu, H. Kim, K.S. Jeong, J. Bae, J.S. Suh, Chem. Mater. 13, 2387 (2001).

[15] A. Burian, J.C. Dore, T. Kyotani, V. Honkimaki, Carbon 43, 2723 (2005).

[16] Q. Yang, W. Xu, A. Tomita, T. Kyotani, J. Am. Chem. Soc. 127, 8956 (2005).

[17] Q. Yang, W. Xu, A. Tomita, T. Kyotani, Chem. Mater. 17, 2940 (2005).

[18] A.P. Hammersley, S.O. Svensson, M. Hanfland, A.N. Fitch, D. Hauserman, High Pressure Res. 14, 235 (1996).

[19] A.P. Hammersley, ESRF Internal Report, ESRF98HA01T, Grenoble 1998.

[20] L. Hawelek, A. Brodka, J.C. Dore, V. Honkimaki, A. Burian, Philos. Mag. 87, 4973 (2007).

[21] L. Hawelek, J. Koloczek, A. Burian, J.C. Dore, V. Honkimaki, T. Kyotani, J. Alloys Comp. 401, $51(2005)$.

[22] A. Brodka, J. Koloczek, A. Burian, J. Nanosci. Nanotechnol. 7, 1505 (2007) 